WUGRAV-95-2

\title{
Gravitational waves from inspiralling compact binaries: Parameter estimation using second-post-Newtonian waveforms
}

\author{
Eric Poisson and Clifford M. Will \\ McDonnell Center for the Space Sciences, Department of Physics, \\ Washington University, St. Louis, Missouri 63130
}

\begin{abstract}
The parameters of inspiralling compact binaries can be estimated using matched filtering of gravitational-waveform templates against the output of laser-interferometric gravitational-wave detectors. The estimates are most sensitive to the accuracy with which the phases of the template and signal waveforms match over the many cycles received in the detector frequency bandwidth. Using a recently calculated formula, accurate to second postNewtonian $(2 \mathrm{PN})$ order [order $(v / c)^{4}$, where $v$ is the orbital velocity], for the frequency sweep $(d F / d t)$ induced by gravitational radiation damping, we study the statistical errors in the determination of such source parameters as the "chirp mass" $\mathcal{M}$, reduced mass $\mu$, and spin parameters $\beta$ and $\sigma$ (related to spin-orbit and spin-spin effects, respectively). We find that previous results using template phasing accurate to $1.5 \mathrm{PN}$ order actually underestimated the errors in $\mathcal{M}, \mu$, and $\beta$. Templates with $2 \mathrm{PN}$ phasing yield somewhat larger measurement errors because the $2 \mathrm{PN}$ corrections act to suppress slightly the importance of spin-orbit contributions to the phase, thereby increasing the measurement error on $\beta$. This, in turn, results in larger measurement errors on $\mathcal{M}$ and $\mu$ because of the strong correlations among the parameters. For two inspiralling neutron stars, the measurement errors increase by less than 16 percent.
\end{abstract}

PACS numbers: 04.25.Nx, 04.30.-w, 97.60.Jd, 97.60.Lf 


\section{INTRODUCTION}

Inspiralling compact binary systems, composed of neutron stars and/or black holes, have been identified [1,2] as the most promising source of gravitational waves for interferometric detectors such as the American LIGO (Laser Interferometer Gravitational-wave Observatory [3]) and the European Virgo [4]. These systems evolve under the influence of gravitational radiation reaction, so that the gravitational-wave signal increases in amplitude as its frequency sweeps through the detector frequency bandwidth, from approximately $10 \mathrm{~Hz}$ to $1000 \mathrm{~Hz}$. (This characteristic signal is often referred to as a "chirp.") Inspiralling compact binaries are especially promising because recent estimates [5, 6] indicate that their event rate could be as large as one hundred per year, for signals detectable out to hundreds of Mpc by the advanced version of LIGO, and because the signal can be accurately predicted using general relativity.

That the signal can be calculated with high accuracy is essential for the measurement of the source parameters [7], which include distance, position in the sky, orientation of the orbital plane, and the masses and spins of the companions. Loosely speaking, the measured signal is passed through a linear filter constructed from the expected signal $h(t ; \boldsymbol{\theta})$ and the spectral density of the detector noise [8] (see below). The signal-to-noise ratio $S / N(\boldsymbol{\theta})$ is then computed. The expected signal and the signal-to-noise ratio are expressed as functions of the vector $\boldsymbol{\theta}$ which collectively represents the source parameters. The actual value of these parameters, which we denote $\tilde{\boldsymbol{\theta}}$, is unknown prior to the measurement. When $\boldsymbol{\theta}=\tilde{\boldsymbol{\theta}}$ the linear filter becomes the Wiener optimum filter which is well known to yield the largest possible signal-to-noise ratio [8]. The source parameters can therefore be determined by maximizing $S / N(\boldsymbol{\theta})$ over a broad collection of expected signals $h(t ; \boldsymbol{\theta})$, loosely referred to as "templates."

The gravitational-wave signal can be characterized by a growing amplitude and a phase which accumulates nonlinearly with time [1]. The signal undergoes a number $N$ of oscillations, varying from 600 to 16000 depending on the nature of the system (see below), as the frequency sweeps through the detector bandwidth. It has been established that it is the phasing of the signal which plays the largest role in parameter estimation [7, 9, 10]. This is because a slight variation in the parameters can quickly cause $h(t ; \boldsymbol{\theta})$ to get out of phase with respect to the true signal $h(t ; \tilde{\boldsymbol{\theta}})$, thus seriously reducing $S / N(\boldsymbol{\theta})$ from its maximum possible value. Therefore a good match between the template's phase and that of the measured signal, throughout the $N$ cycles, singles out, to a large extent, the value of the source parameters. (Clearly, this is only true for those parameters which affect the phasing of the waves, such as the masses and spins of the companions; see below.)

In principle, the gravitational-wave signal from an inspiralling compact binary can be calculated exactly using general relativity (this would require the numerical integration of Einstein's equations). In practice, however, one must rely on some approximation scheme. It appears appropriate, in this context, to adopt a slow-motion approximation [11, and to solve the field equations using a combination of post-Newtonian and post-Minkowskian expansions [12]. To date, the waveform has been calculated accurately through order $(v / c)^{4}$ (where $v$ is the orbital velocity) beyond the leading-order, quadrupole-formula expression 13 [15. Leading-order expressions are referred to as "Newtonian;" the waveform is therefore known to second-post-Newtonian, or $2 \mathrm{PN}$, order. 
The detailed expression for the $2 \mathrm{PN}$ waveform is complicated: the dependence on the various angles (position of the source in the sky, orientation of the detector, orientation of the polarization axes) is not simple, and the waves have several frequency components given by the harmonics of the orbital frequency (assuming that the orbit is circular [16, [17]). For the purpose of this paper, and following Cutler and Flanagan [10], we shall use a simplified expression for the waveform. We shall ignore all post-Newtonian corrections to the wave's amplitude, and single out its dominant frequency component at twice the orbital frequency [1]. Thus, setting $G=c=1$,

$$
h(t ; \boldsymbol{\theta})=r^{-1} Q(\text { angles }) \mathcal{M}(\pi \mathcal{M} F)^{2 / 3} \cos \Phi(t) .
$$

Here, $r$ is the distance to the source, $Q$ a function of the various angles mentioned above, $F(t)$ the gravitational-wave frequency, and $\Phi(t)=\int 2 \pi F(t) d t$ the phase. We have also introduced the (so-called) chirp mass $\mathcal{M}$ : If $\mu=m_{1} m_{2} /\left(m_{1}+m_{2}\right)$ is the reduced mass and $M=m_{1}+m_{2}$ the total mass, then

$$
\mathcal{M}=\eta^{3 / 5} M, \quad \eta=\mu / M .
$$

In Eq. (1.1) we use the most accurate expression available for the phase function $\Phi(t)$. It is determined by the $2 \mathrm{PN}$ expression for the frequency sweep [13],

$$
\begin{aligned}
\frac{d F}{d t}= & \frac{96}{5 \pi \mathcal{M}^{2}}(\pi \mathcal{M} F)^{11 / 3}\left[1-\left(\frac{743}{336}+\frac{11}{4} \eta\right)(\pi M F)^{2 / 3}+(4 \pi-\beta)(\pi M F)\right. \\
& \left.+\left(\frac{34103}{18144}+\frac{13661}{2016} \eta+\frac{59}{18} \eta^{2}+\sigma\right)(\pi M F)^{4 / 3}\right]
\end{aligned}
$$

Apart from the parameters introduced previously, $d F / d t$ also depends on $\hat{\boldsymbol{L}}$, the direction of orbital angular momentum, and on $\boldsymbol{S}_{1}$ and $\boldsymbol{S}_{2}$, the spin angular momentum of each companion. This dependence is hidden in the "spin-orbit" parameter [18]

$$
\beta=\frac{1}{12} \sum_{i=1}^{2}\left[113\left(m_{i} / M\right)^{2}+75 \eta\right] \hat{\boldsymbol{L}} \cdot \boldsymbol{\chi}_{i},
$$

where $\boldsymbol{\chi}_{i}=\boldsymbol{S}_{i} / m_{i}{ }^{2}$, and the "spin-spin" parameter [18]

$$
\sigma=\frac{\eta}{48}\left(-247 \boldsymbol{\chi}_{1} \cdot \boldsymbol{\chi}_{2}+721 \hat{\boldsymbol{L}} \cdot \boldsymbol{\chi}_{1} \hat{\boldsymbol{L}} \cdot \boldsymbol{\chi}_{2}\right) .
$$

The purpose of this paper is to estimate the anticipated accuracy with which the various parameters (such as $\mathcal{M}, \eta, \beta$, and $\sigma$ ) can be determined during a gravitational-wave measurement. This analysis differs from that of Cutler and Flanagan [10] in that it incorporates terms of $2 \mathrm{PN}$ order into the phasing [terms of order $(\pi M F)^{4 / 3}$ in Eq. (1.2)]; their calculations were accurate only through $1.5 \mathrm{PN}$ order (terms of order $\pi M F$ ). Previous analyses also include Refs. 99:19 21].

Our main conclusion is that $1.5 \mathrm{PN}$ phasing underestimates the uncertainty in such parameters as $\mathcal{M}, \eta$, and $\beta$ : $2 \mathrm{PN}$ phasing predicts somewhat larger measurement errors. This is true even when no attempt is made to determine the spin-spin parameter $\sigma$. If, however, $\sigma$ is also estimated, then the measurement errors become even larger. This is because the 
TABLE I. Contributions to the accumulated number of wave cycles measured in a LIGO/Virgo-type detector. The frequency entering the bandwidth is $10 \mathrm{~Hz}$ (seismic limit); the frequency leaving is $1000 \mathrm{~Hz}$ (system A) (shot noise), and $360 \mathrm{~Hz}$ (system B) and $190 \mathrm{~Hz}$ (system C) (innermost circular orbit). The various contributions correspond to various terms in Eq. (1.3). Newtonian: first term within the square brackets; $1 \mathrm{PN}$ : second term; tail: $4 \pi(\pi M F)$; spin-orbit: $-\beta(\pi M F) ; 2 \mathrm{PN}:(\pi M F)^{4 / 3}$ terms with $\sigma=0$; and spin-spin: $\sigma(\pi M F)^{4 / 3}$.

\begin{tabular}{lccc}
\hline \hline system & $\mathrm{A}$ & $\mathrm{B}$ & $\mathrm{C}$ \\
\hline Newtonian & 16050 & 3580 & 600 \\
$1 \mathrm{PN}$ & 439 & 212 & 59 \\
tail & -208 & -180 & -51 \\
spin-orbit & $17 \beta$ & $14 \beta$ & $4 \beta$ \\
2PN & 9 & 10 & 4 \\
spin-spin & $-2 \sigma$ & $-3 \sigma$ & $-\sigma$ \\
\hline \hline
\end{tabular}

number of estimated parameters has increased with respect to the number contained in the 1.5PN waveform.

An independent analysis by Cutler and Flanagan [22] shows that 2PN waveforms are not sufficiently accurate for the purpose of parameter estimation: they produce systematic errors which are larger than the statistical errors inherent to the measurement process. This is because the $2 \mathrm{PN}$ waveform fails to remain in phase with the true general-relativistic signal, even when the source parameters are exactly matched [23 25]. To construct templates such that the systematic errors will fall below the measurement errors will require an expression for the wave's phasing accurate through at least 3PN order [23 25]. To achieve such a high degree of accuracy is a major challenge for gravitational-wave theorists.

We shall consider the following three "canonical" binary systems:

System A: two neutron stars, with $m_{1}=m_{2}=1.4 M_{\odot}$;

System B: neutron star and black hole, with $m_{1}=1.4 M_{\odot}$ (the neutron star) and $m_{2}=10 M_{\odot}$ (the black hole); and

System C: two black holes, with $m_{1}=m_{2}=10 M_{\odot}$.

For each of these systems Table I summarizes the contribution from each term in Eq. (1.3) to the total number of gravitational-wave cycles received in a LIGO/Virgo-type detector.

The remainder of the paper is organized as follows. In Sec. II we review the theory of parameter estimation, as developed in previous papers by Finn [26] and Cutler and Flanagan [10]. In Sec. III we carry out the calculations for the waveform (1.1), and compute the anticipated uncertainty in the measured values of the source parameters. Our results are summarized and discussed in Sec. IV.

\section{PARAMETER ESTIMATION: THEORY}

The theory of detection and measurement of gravitational-wave signals was put on a firm statistical foundation, rather similar to that underlying the theory of radar detection [8,27]. 
This was done by various authors, including Finn [26] and Cutler and Flanagan [10]. In this section we review the various aspects of the theory which are relevant for our purposes.

We assume that some criterion has been applied to conclude that a signal originating from an inspiralling compact binary has been received by a network of gravitational-wave detectors. It is therefore known that a signal of the form $h(t ; \boldsymbol{\theta})$ has passed through the detectors, and we seek to determine the value of the source parameters $\boldsymbol{\theta}$ and the measurement error $\Delta \boldsymbol{\theta}=\boldsymbol{\theta}-\tilde{\boldsymbol{\theta}}$, where $\tilde{\boldsymbol{\theta}}$ denotes the true value.

Finn [26] has derived an expression for $p(\boldsymbol{\theta} \mid s)$, the probability that the gravitational-wave signal is characterized by the parameters $\boldsymbol{\theta}$, given that the detector output is $s(t)$ and that a signal $h(t ; \boldsymbol{\theta})$ — for any value of the parameters $\boldsymbol{\theta}$ — is present. The detector output is given by

$$
s(t)=h(t ; \boldsymbol{\theta})+n(t)
$$

where $n(t)$ represents the detector noise, assumed to be a stationary, Gaussian random process. Finn shows that

$$
p(\boldsymbol{\theta} \mid s) \propto p^{(0)}(\boldsymbol{\theta}) \exp \left[-\frac{1}{2}(h(\boldsymbol{\theta})-s \mid h(\boldsymbol{\theta})-s)\right],
$$

where $p^{(0)}(\boldsymbol{\theta})$ is the a priori probability that the signal is characterized by $\boldsymbol{\theta}$ (this represents our prior information regarding the possible value of the parameters) and where the constant of proportionality is independent of $\boldsymbol{\theta}$.

The inner product $(\cdot \mid \cdot)$ is defined as follows [10]. The statistical properties of the detector noise can be summarized by its autocorrelation function $C_{n}(\tau)=\langle n(t) n(t+\tau)\rangle$, where $\langle\cdot\rangle$ denotes a time average. (It is assumed that the noise has zero mean.) The Fourier transform of the autocorrelation function gives the noise spectral density

$$
S_{n}(f)=2 \int_{-\infty}^{\infty} C_{n}(\tau) e^{2 \pi i f \tau} d \tau
$$

which is defined for $f>0$ only. The inner product is defined so that the probability for the noise $n(t)$ to have a particular realization $n_{0}(t)$ is given by $p\left(n=n_{0}\right) \propto \exp \left[-\left(n_{0} \mid n_{0}\right) / 2\right]$. It is given by

$$
(g \mid h)=2 \int_{0}^{\infty} \frac{\tilde{g}^{*}(f) \tilde{h}(f)+\tilde{g}(f) \tilde{h}^{*}(f)}{S_{n}(f)} d f,
$$

where $\tilde{g}(f)$ is the Fourier transform of $g(t)$,

$$
\tilde{g}(f)=\int_{-\infty}^{\infty} g(t) e^{2 \pi i f t} d t
$$

an asterisk denotes complex conjugation.

We define $\rho$, the signal-to-noise ratio associated with the measurement, to be the norm of the signal $h(t ; \boldsymbol{\theta})$,

$$
\rho^{2}=(h \mid h)=4 \int_{0}^{\infty} \frac{|\tilde{h}(f)|^{2}}{S_{n}(f)} d f
$$


evaluated at $\boldsymbol{\theta}=\tilde{\boldsymbol{\theta}}$, the true value of the source parameters. This is the largest possible value of the signal-to-noise ratio, for $\tilde{h}^{*}(f ; \tilde{\boldsymbol{\theta}}) / S_{n}(f)$ is just the Fourier transform of the Wiener optimum filter [8].

In a given measurement, characterized by the particular detector output $s(t)$, the true value of the source parameters can be estimated by locating the value $\hat{\boldsymbol{\theta}}$ at which the probability distribution function (2.2) is a maximum. This is the so-called maximum-likelihood estimator [8]. In the limit of large signal-to-noise ratio, to which we henceforth specialize, $p(\boldsymbol{\theta} \mid s)$ will be strongly peaked about that value. We now derive a simplified expression for $p(\boldsymbol{\theta} \mid s)$ appropriate for this limiting case.

We first assume that $p^{(0)}(\boldsymbol{\theta})$ is nearly uniform near $\boldsymbol{\theta}=\hat{\boldsymbol{\theta}}$. This indicates that the prior information is practically irrelevant to the determination of the source parameters; we shall relax this assumption below. Then, denoting $\xi(\boldsymbol{\theta})=(h(\boldsymbol{\theta})-s \mid h(\boldsymbol{\theta})-s)$, we have that $\xi$ is minimum at $\boldsymbol{\theta}=\hat{\boldsymbol{\theta}}$. It follows that this can be expanded as

$$
\xi(\boldsymbol{\theta})=\xi(\hat{\boldsymbol{\theta}})+\frac{1}{2} \xi_{, a b}(\hat{\boldsymbol{\theta}}) \Delta \theta^{a} \Delta \theta^{b}+\cdots,
$$

where ", $a$ " denotes partial differentiation with respect to the parameter $\theta^{a}$, and $\Delta \theta^{a}=$ $\theta^{a}-\hat{\theta}^{a}$; summation over repeated indices is understood. We assume that $\rho$ is sufficiently large that the higher-order terms can be neglected. Calculation yields $\xi_{, a b}=\left(h_{, a b} \mid h-s\right)+\left(h_{, a} \mid h_{, b}\right)$, and we assume once more that $\rho$ is large enough that the first term can be neglected (see Cutler and Flanagan [10] for details). We arrive at

$$
p(\boldsymbol{\theta} \mid s) \propto p^{(0)}(\boldsymbol{\theta}) \exp \left[-\frac{1}{2} \Gamma_{a b} \Delta \theta^{a} \Delta \theta^{b}\right]
$$

where

$$
\Gamma_{a b}=\left(h_{, a} \mid h_{, b}\right),
$$

evaluated at $\boldsymbol{\theta}=\hat{\boldsymbol{\theta}}$, is the Fisher information matrix [27].

We therefore see that in the limit of large signal-to-noise ratio, $p(\boldsymbol{\theta} \mid s)$ takes a Gaussian form. From Eq. (2.8) it can be established that the variance-covariance matrix $\Sigma^{a b}$ is given by

$$
\Sigma^{a b} \equiv\left\langle\Delta \theta^{a} \Delta \theta^{b}\right\rangle=\left(\boldsymbol{\Gamma}^{-1}\right)^{a b}
$$

Here, $\langle\cdot\rangle$ denotes an average over the probability distribution function (2.8), and $\Gamma^{-1}$ is the inverse of the Fisher matrix. We define the measurement error in the parameter $\theta^{a}$ to be

$$
\sigma_{a}=\left\langle\left(\Delta \theta^{a}\right)^{2}\right\rangle^{1 / 2}=\sqrt{\sum^{a a}}
$$

(no summation over repeated indices), and the correlation coefficient between parameters $\theta^{a}$ and $\theta^{b}$ as

$$
c^{a b}=\frac{\left\langle\Delta \theta^{a} \Delta \theta^{b}\right\rangle}{\sigma_{a} \sigma_{b}}=\frac{\sum^{a b}}{\sqrt{\sum^{a a} \sum^{b b}}} ;
$$

by definition each $c^{a b}$ must lie in the range $(-1,1)$. 
Cutler and Flanagan [10] have shown that in the limit of large signal-to-noise ratio, Eq. (2.8) is valid even when $p^{(0)}(\boldsymbol{\theta})$ is not uniform near $\boldsymbol{\theta}=\hat{\boldsymbol{\theta}}$. In such cases the prior information plays an important role in the determination of the source parameters. The exponential factor is still peaked at $\boldsymbol{\theta}=\hat{\boldsymbol{\theta}}$, but $\hat{\boldsymbol{\theta}}$ no longer represents the maximum-likelihood estimate, and the full probability distribution function $p(\boldsymbol{\theta} \mid s)$ may not be a Gaussian.

For simplicity, and following Cutler and Flanagan [10], we shall restrict attention to cases such that $p^{(0)}(\boldsymbol{\theta})$ is a Gaussian, given by

$$
p^{(0)}(\boldsymbol{\theta}) \propto \exp \left[-\frac{1}{2} \Gamma_{a b}^{(0)}\left(\theta^{a}-\bar{\theta}^{a}\right)\left(\theta^{b}-\bar{\theta}^{b}\right)\right] .
$$

Then $p(\boldsymbol{\theta} \mid s)$ will also take a Gaussian form, and the new variance-covariance matrix will be given by

$$
\boldsymbol{\Sigma}=\left(\boldsymbol{\Gamma}+\boldsymbol{\Gamma}^{(\mathbf{0})}\right)^{-1}
$$

It should be noted that in general, $p(\boldsymbol{\theta} \mid s)$ will be peaked at a value $\langle\boldsymbol{\theta}\rangle$ which differs both from $\hat{\boldsymbol{\theta}}$ and $\overline{\boldsymbol{\theta}}$.

\section{PARAMETER ESTIMATION: CALCULATIONS}

We proceed with the calculation of the Fisher information matrix, Eq. (2.9), for gravitational-wave signals of the form (1.1), and for gravitational-wave detectors of the LIGO/Virgo type. For such detectors the anticipated noise spectral density can be approximated by the analytic expression [10]

$$
S_{n}(f)=\frac{1}{5} S_{0}\left[\left(f_{0} / f\right)^{4}+2+2\left(f / f_{0}\right)^{2}\right]
$$

where $S_{0}$ is a normalization constant irrelevant for our purposes, and $f_{0}$ the frequency at which $S_{n}(f)$ is minimum; we set $f_{0}=70 \mathrm{~Hz}$, which is appropriate for advanced LIGO sensitivity [3]. To mimic seismic noise we assume that Eq. (3.1) is valid for $f>10 \mathrm{~Hz}$ only, and that $S_{n}(f)=\infty$ for $f<10 \mathrm{~Hz}$.

First, we integrate Eq. (1.3) to obtain expressions for $\Phi(F)$ and $t(F)$, respectively the phase and time as functions of gravitational-wave frequency. (Throughout this section we shall distinguish between $F$, the function of time describing the frequency sweep, and $f$, the Fourier-transform variable.) Expanding in powers of $(\pi M F)^{1 / 3}$ and truncating all expressions to $2 \mathrm{PN}$ order, we obtain

$$
\begin{aligned}
\Phi(F)= & \phi_{c}-\frac{1}{16}(\pi \mathcal{M} F)^{-5 / 3}\left[1+\frac{5}{3}\left(\frac{743}{336}+\frac{11}{4} \eta\right)(\pi M F)^{2 / 3}-\frac{5}{2}(4 \pi-\beta)(\pi M F)\right. \\
& \left.+5\left(\frac{3058673}{1016064}+\frac{5429}{1008} \eta+\frac{617}{144} \eta^{2}-\sigma\right)(\pi M F)^{4 / 3}\right]
\end{aligned}
$$

where $\phi_{c}$ is (formally) the value of $\Phi$ at $F=\infty$, and

$$
\begin{aligned}
t(F)= & t_{c}-\frac{5}{256} \mathcal{M}(\pi \mathcal{M} F)^{-8 / 3}\left[1+\frac{4}{3}\left(\frac{743}{336}+\frac{11}{4} \eta\right)(\pi M F)^{2 / 3}-\frac{8}{5}(4 \pi-\beta)(\pi M F)\right. \\
& \left.+2\left(\frac{3058673}{1016064}+\frac{5429}{1008} \eta+\frac{617}{144} \eta^{2}-\sigma\right)(\pi M F)^{4 / 3}\right]
\end{aligned}
$$


where (formally) $t_{c}=t(\infty)$. Of course, the signal cannot be allowed to reach arbitrarily high frequencies; it must be cut off at a frequency $F=F_{i}$ corresponding to the end of the inspiral. We put $\pi M F_{i}=\left(M / r_{i}\right)^{3 / 2}=6^{-3 / 2} ; r_{i}=6 M$ is the Schwarzschild radius of the innermost circular orbit for a test mass moving in the gravitational field of a mass $M$ [28].

Next, we take the Fourier transform of Eq. (1.1) and calculate $\tilde{h}(f)=\int h(t) e^{2 \pi i f t} d t$. It is sufficient to estimate $\tilde{h}(f)$ using the stationary phase approximation, according to which 29

$$
\int g(t) e^{i \phi(t)} d t \simeq\left[\frac{2 \pi i}{\phi^{\prime \prime}\left(t_{0}\right)}\right]^{1 / 2} g\left(t_{0}\right) e^{i \phi\left(t_{0}\right)}
$$

if $g(t)$ varies slowly near $t=t_{0}$ where the phase has a stationary point: $\phi^{\prime}\left(t_{0}\right)=0$ (a prime denotes differentiation with respect to $t$ ). Substituting Eqs. (1.1) and (3.2) into (3.4), discarding the irrelevant negative-frequency component, and neglecting all post-Newtonian corrections to the amplitude of $\tilde{h}(f)$, we obtain

$$
\tilde{h}(f)=\mathcal{A} f^{-7 / 6} e^{i \psi(f)},
$$

where $\mathcal{A} \propto \mathcal{M}^{5 / 6} Q($ angles $) / r$, and

$$
\begin{aligned}
\psi(f)= & 2 \pi f t_{c}-\phi_{c}-\frac{\pi}{4}+\frac{3}{128}(\pi \mathcal{M} f)^{-5 / 3}\left[1+\frac{20}{9}\left(\frac{743}{336}+\frac{11}{4} \eta\right)(\pi M f)^{2 / 3}\right. \\
& \left.-4(4 \pi-\beta)(\pi M f)+10 \epsilon\left(\frac{3058673}{1016064}+\frac{5429}{1008} \eta+\frac{617}{144} \eta^{2}-\sigma\right)(\pi M f)^{4 / 3}\right] .
\end{aligned}
$$

We have introduced the parameter $\epsilon \equiv 1$. This gives us the freedom, for future use, of removing the $2 \mathrm{PN}$ terms from $\psi(f)$ by setting $\epsilon=0$.

We now substitute Eq. (3.5) into (2.6) and calculate the signal-to-noise ratio. We readily obtain

$$
\rho^{2}=20 \mathcal{A}^{2} S_{0}{ }^{-1} f_{0}{ }^{-4 / 3} I(7)
$$

where the integrals $I(q)$ represent various moments of the noise spectral density:

$$
I(q) \equiv \int_{1 / 7}^{x_{i}} \frac{x^{-q / 3}}{x^{-4}+2+2 x^{2}} d x
$$

where $x_{i}=f_{i} / f_{0}=\left(6^{3 / 2} \pi M f_{0}\right)^{-1}$ is the frequency cutoff.

As the next step toward the computation of the Fisher matrix, we calculate the derivatives of $\tilde{h}(f)$ with respect to the seven parameters

$$
\boldsymbol{\theta}=\left(\ln \mathcal{A}, f_{0} t_{c}, \phi_{c}, \ln \mathcal{M}, \ln \eta, \beta, \sigma\right) .
$$

We obtain

$$
\begin{aligned}
\tilde{h}_{, 1} & =\tilde{h} \\
\tilde{h}_{, 2} & =2 \pi i\left(f / f_{0}\right) \tilde{h}, \\
\tilde{h}_{, 3} & =-i \tilde{h},
\end{aligned}
$$




$$
\begin{aligned}
\tilde{h}_{, 4} & =-\frac{5 i}{128}(\pi \mathcal{M} f)^{-5 / 3}\left(1+A_{4} v^{2}-B_{4} v^{3}+C_{4} v^{4}\right) \tilde{h}, \\
\tilde{h}_{, 5} & =-\frac{i}{96}(\pi \mathcal{M} f)^{-5 / 3}\left(A_{5} v^{2}-B_{5} v^{3}+C_{5} v^{4}\right) \tilde{h}, \\
\tilde{h}_{, 6} & =\frac{3 i}{32} \eta^{-3 / 5}(\pi \mathcal{M} f)^{-2 / 3} \tilde{h}, \\
\tilde{h}_{, 7} & =-\frac{15 i}{64} \eta^{-4 / 5}(\pi \mathcal{M} f)^{-1 / 3} \tilde{h},
\end{aligned}
$$

where $v \equiv(\pi M f)^{1 / 3}$. We also have defined

$$
\begin{aligned}
& A_{4}=\frac{4}{3}\left(\frac{743}{336}+\frac{11}{4} \eta\right) \\
& B_{4}=\frac{8}{5}(4 \pi-\beta), \\
& C_{4}=2 \epsilon\left(\frac{3058673}{1016064}+\frac{5429}{1008} \eta+\frac{617}{144} \eta^{2}-\sigma\right),
\end{aligned}
$$

and

$$
\begin{aligned}
& A_{5}=\frac{743}{168}-\frac{33}{4} \eta, \\
& B_{5}=\frac{27}{5}(4 \pi-\beta), \\
& C_{5}=18 \epsilon\left(\frac{3058673}{1016064}-\frac{5429}{4032} \eta-\frac{617}{96} \eta^{2}-\sigma\right) .
\end{aligned}
$$

Finally, the components of $\boldsymbol{\Gamma}$ can be obtained by evaluating the inner products $\left(h_{, a} \mid h_{, b}\right)$ using Eq. (2.4). The $\Gamma_{a b}$ 's can all be expressed in terms of the parameters $\boldsymbol{\theta}$, the signal-tonoise ratio $\rho$, and the integrals $I(q)$. The expressions are too numerous and lengthy to be displayed here. As illustrating examples, we quote

$$
\Gamma_{1 a}=\delta_{1 a} \rho^{2},
$$

and

$$
\begin{aligned}
\Gamma_{46}= & -\frac{15}{4096} \eta^{-3 / 5}\left(\pi \mathcal{M} f_{0}\right)^{-7 / 3}\left[J(14)+A_{4} J(12)\right. \\
& \left.-B_{4} J(11)+C_{4} J(10)\right] \rho^{2},
\end{aligned}
$$

where $J(q) \equiv I(q) / I(7)$. We note that even though the $\tilde{h}_{, a}$ 's are expressed as truncated post-Newtonian expansions in Eq. (3.10), they must be treated as exact when computing $\boldsymbol{\Gamma}$. This is to ensure that the eigenvalues of the Fisher matrix are always positive definite.

The variance-covariance matrix $\Sigma^{a b}$ can now be obtained from Eq. (2.14), and the measurement errors and correlation coefficients computed from Eqs. (2.11) and (2.12). Before doing so, however, we must first state our assumptions regarding the prior information available on the source parameters.

We take advantage of the fact that the dimensionless spin parameters, $\chi_{1}$ and $\chi_{2}$, must necessarily be smaller than unity. (This upper bound is strict for black holes, but only 
TABLE II. Measurement errors and correlation coefficients for the estimation of six parameters ( $\sigma$ is not estimated), assuming $\rho=10$ and $\beta=0$. The first column indicates whether or not prior information was included in the calculation. The second column gives the value of $\epsilon$, introduced in Eq. (3.6); $\epsilon=1$ represents $2 \mathrm{PN}$ phasing. Then follows, in more suggestive notation, $\sigma_{2} / f_{0}$ (in msec), $\sigma_{3}$ (in radians), $\sigma_{4}, \sigma_{5}, \sigma_{6}, c^{45}, c^{46}$, and $c^{56}$ (all dimensionless).

\begin{tabular}{|c|c|c|c|c|c|c|c|c|c|}
\hline prior & $\epsilon$ & $\Delta t_{c}$ & $\Delta \phi_{c}$ & $\Delta \mathcal{M} / \mathcal{M}$ & $\Delta \eta / \eta$ & $\Delta \beta$ & $c^{\mathcal{M} \eta}$ & $c^{\mathcal{M} \beta}$ & $c^{\eta \beta}$ \\
\hline
\end{tabular}

\begin{tabular}{lccccccccc} 
yes & 1 & 1.07 & 2.94 & $0.036 \%$ & 0.279 & 1.33 & -0.989 & 0.994 & -0.999 \\
no & 1 & 1.08 & 2.97 & $0.037 \%$ & 0.282 & 1.35 & -0.989 & 0.994 & -0.999 \\
no & 0 & 1.13 & 4.09 & $0.034 \%$ & 0.243 & 1.24 & -0.988 & 0.993 & -0.999 \\
no & -1 & 1.16 & 4.96 & $0.032 \%$ & 0.213 & 1.15 & -0.986 & 0.992 & -0.999 \\
\hline \multicolumn{7}{l}{ System B }
\end{tabular}

\begin{tabular}{lccccccccc} 
yes & 1 & 1.72 & 2.27 & $0.218 \%$ & 0.503 & 2.29 & -0.993 & 0.996 & -0.999 \\
no & 1 & 1.76 & 2.32 & $0.226 \%$ & 0.523 & 2.38 & -0.993 & 0.996 & -0.999 \\
no & 0 & 2.04 & 6.24 & $0.191 \%$ & 0.386 & 1.99 & -0.990 & 0.994 & -0.999 \\
no & -1 & 2.20 & 8.68 & $0.171 \%$ & 0.306 & 1.76 & -0.988 & 0.993 & -0.999 \\
\multicolumn{7}{l}{ System C (two black holes): }
\end{tabular}

\begin{tabular}{lrllllllll} 
yes & 1 & 1.50 & 2.19 & $0.54 \%$ & 1.46 & 8.19 & -0.946 & 0.956 & -0.999 \\
no & 1 & 2.40 & 4.99 & $1.96 \%$ & 5.50 & 30.8 & -0.996 & 0.997 & -0.999 \\
no & 0 & 3.53 & 9.27 & $1.42 \%$ & 3.16 & 19.5 & -0.992 & 0.994 & -0.999 \\
no & -1 & 4.01 & 14.7 & $1.21 \%$ & 2.22 & 14.9 & -0.989 & 0.992 & -0.999 \\
\hline \hline
\end{tabular}

approximate for neutron stars.) It follows from Eqs. (1.4) and (1.5) that $|\beta|$ must be smaller than approximately 8.5, and that $|\sigma|$ must be smaller than approximately 5.0. Following Cutler and Flanagan [10], we crudely incorporate this information into our calculations by taking

$$
p^{(0)}(\boldsymbol{\theta}) \propto \exp \left[-\frac{1}{2}(\beta / 8.5)^{2}-\frac{1}{2}(\sigma / 5.0)^{2}\right] .
$$

We consider all other parameters to be unconstrained [30].

\section{RESULTS AND DISCUSSION}

Equation (3.12) implies that the Fisher matrix is block diagonal. The parameter $\theta^{1}=$ $\ln \mathcal{A}$ is therefore entirely uncorrelated with the other parameters, and we find $\sigma_{1}=\Delta \mathcal{A} / \mathcal{A}=$ $1 / \rho, c^{1 a}=0$, in all cases. We shall no longer be concerned with this parameter.

The results concerning the other parameters are displayed in Tables II and III. All calculations were carried out assuming $\rho=10$, and that the companions are spinless, so that $\beta=\sigma=0$.

To obtain the results of Table II we have estimated only six (including $\mathcal{A}$ ) of the seven parameters, leaving $\sigma$ out. In effect, we have truncated the original Fisher matrix to a 
smaller, $6 \times 6$, matrix. This amounts to assuming before measurement that the spin-spin parameter must be very small; equivalently, this assumption can be implemented by taking $p^{(0)}(\sigma)$ to be very strongly peaked at $\sigma=0$.

For each of the three canonical systems, the first line of Table II displays the measurement errors and correlation coefficients as calculated using $2 \mathrm{PN}$ phasing $(\epsilon=1)$ and the prior probability distribution function (3.15). The second line shows the same quantities calculated without utilizing the prior information. We notice that the prior information makes virtually no difference for systems A and B, but is very significant for system C.

The third line of Table II displays the measurement errors and correlation coefficients assuming no prior information and $1.5 \mathrm{PN}$ phasing $(\epsilon=0)$. Our values agree with those of Cutler and Flanagan [10,31]. We notice that the errors calculated using 1.5PN phasing are always larger for $t_{c}$ and $\phi_{c}$, and smaller for $\mathcal{M}, \eta$, and $\beta$, than those calculated using $2 \mathrm{PN}$ phasing. Thus, the measurement errors on the masses and spins are underestimated when evaluated using the less accurate $1.5 \mathrm{PN}$ phasing.

This can be explained with a simple argument. In Eq. (3.6), the $1 \mathrm{PN}$ and $2 \mathrm{PN}$ terms [of order $(\pi M f)^{2 / 3}$ and $(\pi M f)^{4 / 3}$ respectively] combine, when $\epsilon=1$, so as to reduce the relative importance of the $\pi M f$ term, when compared to the situation when $\epsilon=0$. In other words, the relative contribution to the total number of wave cycles coming from the $\pi M f$ term is less for $2 \mathrm{PN}$ phasing than it is for $1.5 \mathrm{PN}$ phasing (see Table I). It is therefore expected that $2 \mathrm{PN}$ phasing will produce larger measurement errors for $\beta$, since all information about $\beta$ comes from the $\pi M f$ term. But because $\beta$ is strongly correlated with both $\mathcal{M}$ and $\eta$, it follows that these parameters will also come with larger measurement errors. This is indeed what is observed. It is amusing to test this explanation by artificially setting $\epsilon=-1$ in our calculations, which we do in the fourth line of Table II. The argument suggests that the errors in $\mathcal{M}, \eta$, and $\beta$ should all decrease with respect to the values calculated using $1.5 \mathrm{PN}$ phasing, since the relative importance of the $\pi M f$ term is now increased. This is indeed what the results show.

To obtain the results of Table III we have estimated all seven parameters, including both $\mathcal{A}$ and $\sigma$, and used $2 \mathrm{PN}$ phasing. For each of the three systems, the first line of Table III displays the measurement errors and correlation coefficients calculated using the prior probability distribution function (3.15). We notice that the measurement errors are all significantly larger than those displayed in Table II; this is expected from the fact that we are now estimating a larger number of parameters.

It is interesting to ask how the measurement errors increase as the number of estimated parameters increases. In Ref. [10], Cutler and Flanagan initially estimate only five of their six parameters, leaving $\beta$ out. When they next include $\beta$ in their calculations, they find that the measurement errors on $\mathcal{M}$ and $\mu$ increase by a factor of order 10 . In this paper, on the other hand, we have initially estimated only six of our seven parameters, leaving $\sigma$ out. When we next include $\sigma$ in our calculations, we find that the measurement errors on $\mathcal{M}, \mu$, and $\beta$ only increase by a factor of order unity. Thus, the inclusion of $\sigma$ in the calculation has less dramatic consequences than the inclusion of $\beta$. This confirms a conjecture formulated by Cutler and Flanagan [10] at the end of their Sec. III. That this is so is largely due to the importance of prior information in the estimation of $\sigma$.

In the second line of Table III we display the results obtained when the prior information is not included in the calculations. We notice that for all systems, the prior information 
TABLE III. Measurement errors and correlation coefficients for the estimation of all seven parameters, assuming $\rho=10, \beta=0$, and $\sigma=0$. The first column indicates whether or not prior information was included in the calculation. The notation is similar to that of Table II, and $\epsilon=1$ in all cases.

\begin{tabular}{|c|c|c|c|c|c|c|}
\hline prior & $\Delta t_{c}$ & $\Delta \phi_{c}$ & $\Delta \mathcal{M} / \mathcal{M}$ & $\Delta \eta /$ & $\Lambda$ & $c^{\beta \sigma}$ \\
\hline
\end{tabular}

System A (two neutron stars):

$\begin{array}{lllllllllllll}\text { yes } & 1.28 & 13.3 & 0.047 \% & 0.507 & 1.77 & 4.79 & -0.956 & 0.996 & -0.648 & -0.964 & 0.835 & -0.660\end{array}$

\begin{tabular}{llllllllllllll} 
no & 2.72 & 46.9 & $0.120 \%$ & 1.578 & 4.53 & 17.3 & -0.991 & 0.999 & -0.952 & -0.993 & 0.984 & -0.955 \\
\hline
\end{tabular}

System B (neutron star and black hole):

\begin{tabular}{lrlllrllllllll} 
yes & 2.54 & 23.6 & 0.280 & $\%$ & 0.873 & 3.02 & 4.74 & -0.959 & 0.997 & -0.630 & -0.969 & 0.817 & -0.650 \\
no & 7.52 & 95.9 & $0.813 \%$ & 3.10 & 8.98 & 19.4 & -0.993 & 0.999 & -0.961 & 0.995 & 0.986 & -0.964 \\
\hline
\end{tabular}

System C (two black holes):

\begin{tabular}{lrrrrrrrrrrrr} 
yes & 2.22 & 10.4 & $0.55 \%$ & 1.51 & 8.22 & 4.81 & -0.849 & 0.920 & 0.191 & -0.984 & 0.257 & -0.081 \\
no & 17.0 & 179 & $7.23 \%$ & 30.7 & 149 & 74.6 & -0.995 & 0.998 & -0.962 & -0.999 & 0.984 & -0.978 \\
\hline \hline
\end{tabular}

indeed plays a very important role. In fact, we see that Eq. (3.15) provides nearly all of the information regarding the spin-spin parameter $\sigma$. This explains why the measurement error on $\sigma$ is always nearly equal to 5.0, and its correlation coefficient with other parameters significantly smaller than unity. Of course, these results only apply to gravitational-wave measurements with $\rho=10$. To bring the error on $\sigma$ well below the a priori constraint $\sigma<|5.0|$, say $\Delta \sigma \lesssim 3$, the measurement would require a signal-to-noise ratio larger than approximately 45 for system A, 50 for system B, and 110 for system C.

We conclude with the following remark. It is clear that the results displayed in Tables II and III depend on a fairly large number of simplifying assumptions, and that a more careful treatment might produce somewhat different numbers. These assumptions include: (i) the simplified form (1.1) for the waveform; (ii) the neglect of (not yet calculated) higher-order terms in the post-Newtonian expansion (1.3); (iii) the neglect of $O(1 \rho)$ corrections in the expression (2.8), 2.9) for $p(\boldsymbol{\theta} \mid s)$; (iv) the analytic model (3.1) for the noise spectral density; and (v) our rather crude incorporation of the prior information. We shall leave for future work the difficult task of carefully examining the effect of these assumptions on our results.

\section{ACKNOWLEDGMENTS}

Our warmest thanks to Eanna Flanagan for many useful conversations and his detailed comments on the manuscript. This work was supported by the National Science Foundation under Grant No. PHY 92-22902 and the National Aeronautics and Space Administration under Grant No. NAGW 3874. 


\section{REFERENCES}

[1] K.S. Thorne, in 300 Years of Gravitation, edited by S.W. Hawking and W. Israel (Cambridge University Press, Cambridge, England, 1987), p. 330.

[2] B.F. Schutz, Nature 323, 310 (1986); Class. Quantum Grav. 6, 1761 (1989).

[3] A. Abramovici, W.E. Althouse, R.W.P. Drever, Y. Gürsel, S. Kawamura, F.J. Raab, D. Shoemaker, L. Siewers, R.E. Spero, K.S. Thorne, R.E. Vogt, R. Weiss, S.E. Whitcomb, and M.E. Zucker, Science 256, 325 (1992).

[4] C. Bradaschia, E. Calloni, M. Cobal, R. Del Fasbro, A. Di Virgilio, A. Giazotto L.E. Holloway, H. Kautzky, B. Michelozzi, V. Montelatici, D. Pascuello, and W. Velloso, in Gravitation 1990, Proceedings of the Banff Summer Institute, edited by R. Mann and P. Wesson (World Scientific, Singapore, 1991).

[5] R. Narayan, T. Piran, and A. Shemi, Astrophys. J. 379, L17 (1991).

[6] E.S. Phinney, Astrophys. J. 380, L17 (1991).

[7] C. Cutler, T.A. Apostolatos, L. Bildsten, L.S. Finn, E.E. Flanagan, D. Kennefick, D.M. Markovic, A. Ori, E. Poisson, G.J. Sussman, and K.S. Thorne, Phys. Rev. Lett. 70, 2984 (1993).

[8] L.A. Wainstein and V.D. Zubakov, Extraction of Signals from Noise, (Prentice-Hall, Englewood Cliffs, 1962).

[9] L.S. Finn and D.F. Chernoff Phys. Rev. D 47, 2198 (1993).

[10] C. Cutler and E.E. Flanagan, Phys. Rev. D 49, 2658 (1994).

[11] For an overview, see C.M. Will, in Relativistic Cosmology, Proceedings of the Eighth Nishinomiya-Yukawa Memorial Symposium, edited by M. Sasaki (Universal Academy Press, Kyoto, Japan, 1994), p. 83.

[12] L. Blanchet and T. Damour, Philos. Trans. R. Soc. London A320, 379 (1986); Phys. Rev. D 37, 1410 (1988); Ann. Inst. H. Poincaré 50, 377 (1989); Phys. Rev. D 46, 4304 (1992).

[13] L. Blanchet, T. Damour, B.R. Iyer, C.M. Will, and A.G. Wiseman, Gravitationalradiation damping of compact binary systems to second post-Newtonian order, Phys. Rev. Lett. (in press).

[14] L. Blanchet, T. Damour, and B.R. Iyer, Gravitational waves from inspiralling compact binaries: Energy loss and waveform to second-post-Newtonian order (unpublished).

[15] C.M. Will and A.G. Wiseman, in preparation.

[16] P.C. Peters, Phys. Rev. 136, B1224 (1964).

[17] C.W. Lincoln and C.M. Will, Phys. Rev. D 42, 1123 (1990).

[18] L.E. Kidder, C.M. Will, and A.G. Wiseman, Phys. Rev. D 47, R4183 (1993).

[19] A. Krolak, J.A. Lobo, and B.J. Meers, Phys. Rev. D 48, 3451 (1993).

[20] P. Joranowski and A. Krolak, Phys. Rev. D 49, 1723 (1994).

[21] K. Kokkotas, A. Krolak, and G. Tsegas, Class. Quantum Grav. 11, 1901 (1994); 2833 (1994).

[22] C. Cutler and E.E. Flanagan, in preparation.

[23] C. Cutler, L.S. Finn, E. Poisson, and G.J. Sussman, Phys. Rev. D 47, 1511 (1993).

[24] H. Tagoshi and T. Nakamura, Phys. Rev. D 494016 (1994).

[25] H. Tagoshi and M. Sasaki, Prog. Theor. Phys. 92, 745 (1994).

[26] L.S. Finn, Phys. Rev. D 46, 5236 (1992). 
[27] C.W. Helstrom, Statistical Theory of Signal Detection, (Pergamon, Oxford, England, 1968).

[28] Strictly speaking, our expression for $F_{i}$ is only valid in the limit $\eta \rightarrow 0$. For simplicity, and because this will not affect our results significantly, we shall ignore the corrections to the innermost circular orbit which are due to the finite value of the mass ratio. These are computed by L.E. Kidder, C.M. Will, and A.G. Wiseman, Phys. Rev. D 47, 3281 (1993) using post-Newtonian theory, and by S. Detweiler and J.K. Blackburn, Phys. Rev. D 46, 2318 (1992) and G.B. Cook, Phys. Rev. D 50, 5025 (1994) using numerical relativity.

[29] See, for example, J.D. Jackson, Classical Electrodynamics, (Wiley, New York, 1975), p. 316.

[30] Our calculations should take into account the fact that $\eta$ is restricted to the interval $(0,1 / 4]$. However, this information cannot be incorporated using a Gaussian probability distribution function such as Eq. (3.15). To keep the analysis simple we have preferred to leave $\eta$ unrestricted.

[31] Cutler and Flanagan use $\ln \mu$ as a parameter instead of $\ln \eta$. It is easy to show, by performing the coordinate transformation, that $\Delta \mu / \mu=(2 / 5) \Delta \eta / \eta$ and $c^{\mu a}=c^{\eta a}$. 